\title{
THE RELATION OF PERFECT SETS OF MEASURE ZERO TO CERTAIN CLASSES OF FUNCTIONS
}

\author{
PHILIP T. MAKER
}

1. Introduction. A real-valued function of a real variable defined on a set $A$ is said to satisfy the (N) condition on $A$ if the image set $f(E)$ of every set $E$ of measure zero in $A$ is of measure zero. Lusin has shown* that, when $A$ is an interval and the function is continuous, the (N) condition is satisfied when this property holds on every perfect set of measure zero in the interval. In this paper we extend this result to a much wider class of functions and point out two consequences. The first concerns a generalization of a theorem due to Rademacher $\dagger$ which states that the $(\mathrm{N})$ condition is necessary and sufficient in order that a continuous function transform measurable sets into measurable sets. The second gives a condition necessary and sufficient for the uniform convergence to an absolutely continuous function of certain sequences of absolutely continuous functions. In the last section a covering property of every perfect set of measure zero is pointed out.

We shall denote the Lebesgue measure of $E$ by $m E$ and its outer measure by $|E|$.

2. The $(\mathrm{N})$ condition. We prove the following theorem:

TheOREM 1. Let $f(x)$ be defined on $A$ and satisfy the conditions:

(1) the image of any portion $\ddagger$ of $A$ with respect to $f(x)$ is measurable, and

(2) the set of solutions $x$ of the equation $y=f(x)$ is a closed set with respect to $A$, for all values of $y$ in $(-\infty, \infty)$ except at most for a set of measure zero.

Let $E \subset A$ with $m E=0$ and $P \subset A, m P=0$, and $P$ perfect with respect to $A$. Then $m f(P)=0$ for all $P$ implies $m f(E)=0$.

Proof. Assuming that a set $E$ exists for which $m(E)=0$, and $|f(E)|=k>0$, we let $E$ be covered by a set of intervals $B_{1}$ with $m B_{1}<1 / 2$. Let $C_{1}$ be a finite number of these intervals for which $\left|f\left(C_{1} \cdot E\right)\right|>k / 2$. In a similar manner cover $C_{1} \cdot E$ by $B_{2}$ with $B_{2} \subset C_{1}$ and $m B_{2}<1 / 4$, and let $C_{2}$ be a finite number of the intervals of $B_{2}$ for which $\left|f\left(C_{2} \cdot E\right)\right|>k / 2$. Continuing this process, obtain $C_{i} \subset C_{i-1}$

* N. Lusin, Intégrale et Série Trigonométrique, Moscow, 1915, p. 109 (in Russian).

$\dagger$ H. Rademacher, Monatshefte für Mathematik und Physik, vol. 27 (1916), pp. 183-291.

$\ddagger$ A portion of a set is the intersection of the set and an interval. 
for which $m C_{i}<1 / 2^{i}$ and $\left|f\left(C_{i} \cdot E\right)\right|>k / 2$. Denote by $C$ the set $C_{1} \cdot C_{2} \ldots$, which is closed with respect to $A$, and by $f^{*}(C)$ the set $f\left(C_{1}\right) \cdot f\left(C_{2}\right) \cdots$. Since the measure of $f\left(C_{i}\right)$ is greater than $k / 2$ for all $i$, and $f\left(C_{i}\right) \subset f\left(C_{i-1}\right)$, we have $\lim _{i \rightarrow \infty} m f\left(C_{i}\right)=m \lim _{i \rightarrow \infty} f\left(C_{i}\right)$ $=m f^{*}(C) \geqq k / 2$. If $y_{0}$ is any point in $f^{*}(C)$, either $y_{0}=f\left(x_{0}\right)$, where $x_{0} \varepsilon C_{i}$ for all $i$ and hence $x_{0} \varepsilon C$, in which case $y_{0} \varepsilon f(C)$, or else $y_{0}=f\left(x_{i}\right),\left(x_{i} \varepsilon C_{i}\right)$, an infinite number of the points $x_{i}$ being distinct. By the condition (2) of the theorem, the set of points $y$ of this nature not in $f(C)$ is of measure zero. Hence a subset of $f^{*}(C)$ of positive measure belongs to $f(C)$. Since $C$ differs from a set $P$, perfect with respect to $A$, by a denumerable set, $f(P)$ has positive measure, contrary to hypothesis; hence the proof is complete.

This theorem is generalized to functions defined in a euclidean $m$-dimensional space by an obvious modification of the proof.

Condition (1) holds for every function of Baire's classification, defined on a closed set $F$, since for these functions the image of any portion of $F$ is analytic and hence measurable. $\dagger$ This permits us to state and prove the following generalization of the theorem of Rademacher already cited :

THEOREM 2. Let $f(x)$ be a function of Baire's classification defined on the closed set $F$ and satisfying the condition (2) of Theorem 1. Then $f(x)$ transforms measurable sets into measurable sets if and only if, for every perfect set $P$ of measure zero in $F, m f(P)=0$.

Proof. The condition is necessary, since if for some $P \subset F$ we have $m f(P)=k>0$, then there exists a subset $\bar{P}$ of $P$ for which $f(\bar{P})$ is nonmeasurable. But this is contrary to the conclusion of the theorem.

To prove the sufficiency, note that any measurable set $E$ is the sum of an $F_{\sigma}$ and a set $E_{0}$ of measure zero. Hence $f(E)=f\left(F_{\sigma}\right)+f\left(E_{0}\right)$. Since $f\left(F_{\sigma}\right)$ is an analytic set, and $f\left(E_{0}\right)$, by Theorem 1 , is of measure zero, $f(E)$ is measurable.

Condition (2) obviously holds for functions defined and continuous on a closed set and for functions of class $T_{1}$, that is, for any function that takes each of its values, except for those of a set of measure zero, only a finite number of times. This class includes all functions defined on an interval which are of bounded variation, since for these it is known $\ddagger$ that the function $N_{f}(y)$, equal to the number of roots of the equation $y=f(x)$, is summable for $-\infty<y<\infty$. Since, in addition,

$\dagger$ N. Lusin, Lȩ̧ons sur les Ensembles Analytiques, Paris, 1930, p. 152.

$\ddagger$ H. Kestelman, Journal of the London Mathematical Society, vol. 9 (1934), p. 167. 
functions of bounded variation are in Baire's first class, Theorems 1 and 2 apply to them.

3. Sequences of absolutely continuous functions. We prove the following theorem:

THEOREM 3. If the sequence of absolutely continuous functions $f_{n}(x)$, $(n=1,2, \cdots)$, defined in the interval $(a, b)$, converges to $f(x)$ of bounded variation, then the convergence is uniform and $f(x)$ is absolutely continuous if and only if, for every perfect set $P$ of measure zero, the closure of $\sum_{n=1}^{\infty} f_{n}(P)$ is of measure zero.

Proof. If the sequence is not uniformly convergent, the functions $f_{n}(x)$ are not equally continuous. In this case there exist an $\epsilon$, a subsequence $\left\{f_{n_{i}}(x)\right\}$, and the intervals $\left(x_{i}^{\prime}, x_{i}^{\prime \prime}\right)$, such that $\left|f_{n_{i}}\left(x_{i}^{\prime}\right)-f_{n_{i}}\left(x_{i}^{\prime \prime}\right)\right|>\epsilon$, and $\left|x_{i}^{\prime}-x_{i}^{\prime \prime}\right|<1 / 2^{i}$. These intervals $\left(x_{i}^{\prime}, x_{i}^{\prime \prime}\right)$ have a limit point $x_{0}$, and $\left|f_{n}\left(x_{0}\right)-f\left(x_{0}\right)\right|<\epsilon / 4$, for $n>n_{0}$. It is readily verified that in every neighborhood of $x_{0}$ an infinite number of functions take all values in the interval $I=\left[f\left(x_{0}\right)+\epsilon / 4, f\left(x_{0}\right)+\epsilon / 2\right]$, or in the interval $\left[f\left(x_{0}\right)-\epsilon / 2, f\left(x_{0}\right)-\epsilon / 4\right]$ or both, on the $y$ axis. To fix the ideas, assume that each member of the sequence $\bar{f}_{j}(x),(j=1,2, \cdots)$, takes all values in $I$ within the given neighborhood of $x_{0}$. Let $\left\{r_{n}\right\}$ be some enumeration of the rational numbers in $I$. The points $\left\{x_{n}\right\}$ exist such that $\bar{f}_{j_{n}}\left(x_{n}\right)=r_{n}$ and $\left|x_{n}-x_{0}\right|<\left|x_{n-1}-x_{0}\right|$. This last condition allows the points $\left\{x_{n}\right\}$ to be embedded in a perfect set $P$ of measure zero for which the closure of $\sum_{n=1}^{\infty} f_{n}(P)$ will contain $I$, contrary to the hypothesis that the closure is of measure zero. The convergence is therefore uniform; hence the limit function $f(x)$ is continuous. We have only to show that $f(x)$ satisfies the $(\mathrm{N})$ condition.*

Since for every perfect set $\mathrm{P}$ of measure zero the closure of $\sum_{n=1}^{\infty} f_{n}(P)$ is of measure zero, the subset $f(P)$ is also of measure zero, and by Theorem 1 the $(\mathrm{N})$ condition is satisfied.

To prove the condition necessary, suppose $f(x)$ absolutely continuous; then if $P$ is any perfect set of measure zero, $f(P)$ is closed and of measure zero. For a given $\epsilon$ let $f(P)$ be covered by a finite number of intervals, the sum of their lengths being less than $\epsilon / 3$. Let the number of such intervals used be $N$. Lengthen each of these intervals at each end by a length $\epsilon / 6 N$. By the uniform convergence of the sequence, $k$ exists so that $\left|f_{n}(x)-f(x)\right|<\epsilon / 6 N$ for $n>k$. Hence the points $\sum_{n=k}^{\infty} f_{n}(P)$ will be included by the newly lengthened intervals. Since $\sum_{n=1}^{k-1} f_{n}(P)$ is closed and of measure zero, let it be covered by a

* S. Banach, Fundamenta Mathematicae, vol. 7 (1925), pp. 225-237. 
finite set of intervals the sum of the lengths of which is less than $\epsilon / 3$. This being done, $\sum_{n=1}^{\infty} f_{n}(P)$ is covered by a finite number of intervals of length less than $\epsilon$, and the proof is complete.

From this result and a theorem of Helly* which states that in a sequence of uniformly bounded functions $\left\{f_{n}(x)\right\},(a \leqq x \leqq b)$, of uniformly bounded variation there is a subsequence $\left\{f_{n_{k}}(x)\right\}$ converging everywhere to a function of bounded variation, we obtain the following theorem on the compactness of certain sets of absolutely continuous functions:

THEOREM 4. Let $\mathcal{F}$ be a family of uniformly bounded, absolutely continuous functions of uniformly bounded variation with a common interval of definition $(a, b)$. A necessary and sufficient condition that $\mathcal{F}$ contain a sequence converging uniformly to an absolutely continuous function is that, for some sequence $\left\{f_{n}(x)\right\}$ in $\mathcal{F}$, the closure of $\sum_{n=1}^{\infty} f_{n}(P)$ be of measure zero for every perfect set $P$ of measure zero in $(a, b)$.

4. A property of perfect sets of measure zero. Any bounded, perfect set of measure zero can be covered by a finite number of intervals, the sum of the lengths of these being arbitrarily small. The following theorem shows that this covering is not possible, even with an infinite sequence $\left\{I_{n}\right\}$ of intervals, if the length of $I_{n}$ diminishes sufficiently fast as $n$ increases.

THEOREM 5. For every non-empty perfect set $P$ of measure zero there is a sequence of positive numbers $l_{n}$ such that no sequence of intervals with lengths $l_{n}$ will contain $P$.

Proof. With no loss of generality we assume $P$ given in the interval $(a, b)$ with end points $a$ and $b$. There exists a continuous, monotone, but non-absolutely continuous function $f(x)$ defined on $(a, b)$ with $f(a)=0$ and $f(b)=1$ which "grows" only on $P$. $\dagger$ Hence $m f(P)=1$. Since $f(x)$ is uniformly continuous in the closed interval $(a, b), l_{n}$ exists such that in every interval of length $l_{n}$ the oscillation of $f(x)$ is less than $1 / 2^{n}$ for $n=1,2, \cdots$. If the theorem is false, it is possible to cover $P$ with a sequence of intervals $\left\{I_{n}\right\}$ such that the length of $I_{n}$ is $l_{n}$ and therefore such that the sum of the oscillations of $f(x)$ on the intervals $I_{n}$ will be less than 1 . Since $f(x)$ is monotone and continuous, the oscillation of $f(x)$ on $I_{n}$ is $m f\left(I_{n}\right)$. Hence $1>\sum m f\left(I_{n}\right) \geqq m f(P)=1$, which is impossible, and the proof is complete.

UNIVERSITY OF ILLINOIS

* A. Zygmund, Trigonometrical Series, Warsaw, 1935, p. 80.

† S. Saks, Theory of the Integral, New York, 1937, p. 101. 